\title{
Expansion formulae for the homogenised determinant of anisotropic checkerboards
}

\author{
By Marc Briane ${ }^{1}$ and Yves CapdeboscQ ${ }^{2}$ \\ ${ }^{1}$ Centre de Mathématiques, I.N.S.A. de Rennes \& I.R.M.A.R., 20 avenue des \\ Buttes de Coësmes, 35043 Rennes cedex, France.(mbriane@insa-rennes.fr) \\ ${ }^{2}$ Laboratoire de Mathématiques de Versailles, U.V.S.Q., 45 avenue des \\ États-Unis, 78035 Versailles cedex, France.(ycrc@math.uvsq.fr)
}

In this paper some effective properties of anisotropic four-phase periodic checkerboards are studied in two-dimensional electrostatics. An explicit low-contrast secondorder expansion for the determinant of the effective conductivity is given. In the case of a two-phase checkerboard with commuting conductivities the expansion reduces to an explicit formula for the effective determinant (valid for any contrast) as soon as the second-order term vanishes. Such an explicit formula cannot be extended to four-phase checkerboards. A counter-example with high-contrast conductivities is provided. The construction of the counter-example is based on a factorisation principle, due to Astala \& Nesi, which allows us to pass from an anisotropic four-phase square checkerboard to an isotropic one with the same effective determinant.

Keywords: homogenisation, checkerboards, explicit anisotropic formulae

\section{Introduction}

This paper deals with the effective properties of composites with varying conductivity in two-dimensional electrostatics, in the special case where the conductivity is periodic and where the pattern reproduced periodically is a square. Consider the following conduction problem in a bounded domain $\Omega$ of $\mathbb{R}^{2}$ :

$$
-\operatorname{div}\left(A_{\varepsilon} \nabla u_{\varepsilon}\right)=f \text { in } \Omega, \quad u_{\varepsilon}=0 \text { on } \partial \Omega .
$$

The solution $u_{\varepsilon}$ is the voltage potential and $f$ is the density of electric charges. The conductivity $A_{\varepsilon}(\cdot)$ is a highly oscillating sequence of the form $A(\cdot / \varepsilon)$ where $A$ is a $(0,1)^{2}$-periodic matrix-valued function. Then, the effective (constant) matrix $A^{*}$ is the conductivity of the asymptotic problem satisfied by the limit potential as $\varepsilon$ tends to zero (see formula (2.2) and Definition 3.1). We specialise to the case where each square cell is composed of four anisotropic phases:

$$
\begin{array}{|l|l|}
\hline A_{1} & A_{2} \\
\hline A_{4} & A_{3} \\
\hline
\end{array}
$$

The conductivity $A$ is constant in each phase of value $A_{i}$. Since $A$ may be anisotropic, each $A_{i}$ is a two by two symmetric positive definite matrix. From a mathematical point of view, the effective properties are deduced from the $\varepsilon$-rescaled 
periodic microstructure as the period $\varepsilon$ tends to zero through a homogenisation process (see e.g. Bensoussan et al. 1978 for an introduction of the homogenisation theory). Our aim is to obtain explicit formulae involving the effective or homogenised matrix. In fact, we restrict ourselves to one coefficient: the determinant of the effective matrix (simply called the effective or homogenised determinant in the sequel).

The effective properties of periodic composites have been widely studied, especially in the case of two-dimensional two-phase composites. After the seminal works of Rayleigh (1892) and Maxwell (1904), Keller (1964) introduced a duality method in order to characterise the effective properties of such composites. His work was extended by Dykhne (1970), Mendelson (1975), and more recently by Golden \& Milton (1990). Moreover, explicit solutions were obtained by Berdichevski (1985) and Obnosov (1999) for checkerboard structures, and by Mityushev (1995) for cylindrical inclusions. As an alternative to the derivation of explicit formulae, there is a considerable amount of works on the bounds for, or the approximation of effective coefficients; we refer to Milton (2002) (and the references therein) for a quite complete review on the bounds theory. On the other hand, numerical results for the effective conductivity of checkerboards were also obtained by Torquato et al. (1999) and by Michel et al. (1999). One of the motivations for deriving explicit effective coefficients is for the validation of the numerical approaches.

There are very few explicit formulae for effective coefficients for periodic composites. In particular, there is one result for four-phase structures, that explicitly yields the effective coefficients of an isotropic four-phase square checkerboard. In this isotropic setting, each conductivity $A_{i}$ is equal to $a_{i} I_{2}$ where $a_{i}>0$ and $I_{2}$ is the identity matrix of $\mathbb{R}^{2 \times 2}$. The formula was conjectured by Mortola \& Steffé (1985), and was proved fifteen years later by Craster \& Obnosov (2001) for a rectangular checkerboard and independently by Milton (2001) for a square checkerboard. In the case of a four-phase rectangular checkerboard:

\begin{tabular}{|l|l|}
\hline$a_{1}$ & $a_{2}$ \\
\hline$a_{4}$ & $a_{3}$ \\
\hline
\end{tabular}

the formula of the effective matrix $A^{*}$ is rather complicated, but its determinant is given by the following formula

$$
\operatorname{det} A^{*}=\frac{a_{2} a_{3} a_{4}+a_{1} a_{3} a_{4}+a_{1} a_{2} a_{4}+a_{1} a_{2} a_{3}}{a_{1}+a_{2}+a_{3}+a_{4}} .
$$

The simplicity of this formula illustrates that, amongst two-dimensional effective constants, the determinant presents particular properties. There are other results that specifically involve the effective determinant. For example, it is known (see e.g. Francfort \& Murat 1987) that the conductivity matrix of any two-dimensional microstructure (possibly non-periodic) with a constant determinant, induces by homogenisation an effective conductivity matrix with the same determinant.

Taking into account the previous results and remarks, we tried to extend the determinant formula (1.1) to an anisotropic four-phase square checkerboard. We did not find a general explicit formula of the effective determinant for such an anisotropic composite. However, assuming that the four phases $A_{i}$ of the checkerboard admit a second-order expansion around a given matrix $A_{0}$, we obtain an explicit second-order expansion for the effective determinant only in terms of $A_{i}$ 
and $A_{0}$ :

$$
\operatorname{det} A^{*}=D^{*}-F\left(A_{0}\right) E^{*}+o\left(\delta^{2}\right),
$$

where $A^{*}$ is the effective matrix of the checkerboard, $D^{*}$ and $E^{*}$ are explicit functions of the four phases, and $F\left(A_{0}\right)>0$ is an explicit function of the reference matrix $A_{0}$.

In the case of an anisotropic two-phase checkerboard with commuting conductivity matrices $\left(A_{3}=A_{1}\right.$ and $A_{4}=A_{2}$ with $\left.A_{1} A_{2}=A_{2} A_{1}\right)$, we prove that the expansion (1.2) gives the exact effective determinant, i.e., $\operatorname{det} A^{*}=D^{*}$, if the second-order term $E^{*}$ is zero. This leads to the explicit formula $\operatorname{det} A^{*}=\sqrt{\operatorname{det}\left(A_{1} A_{2}\right)}$.

The situation is much more delicate in the case of an anisotropic four-phase checkerboard. We then restrict ourselves to diagonal conductivity matrices. In section 3 we introduce a stability property which connects the effective determinant of the checkerboard with phases $A_{i}$ to the one with phases $B A_{i} B$, for any positive definite diagonal matrix $B$. Using the Craster-Obnosov formula (Craster \& Obnosov 2001) we check that any isotropic four-phase checkerboard satisfies the stability property. Assuming this property for an anisotropic four-phase checkerboard leads to the expansion (1.2). But in contrast to the case of two-phase checkerboards, the condition $E^{*}=0$ does not imply that the correct effective determinant is obtained, i.e., in general $\operatorname{det} A^{*} \neq D^{*}$.

To prove this negative result, we build an anisotropic four-phase square checkerboard with high-contrast conductivities, which both satisfies $E^{*}=0$ and $\operatorname{det} A^{*} \neq$ $D^{*}$. The counter-example is based on a nice factorisation principle due to Astala \& Nesi (see Section 5). This principle allows us to deduce an anisotropic four-phase square checkerboard from an irregular but isotropic one with the same determinant.

The paper is organised as follows. In Section 2 we prove an explicit expansion of the effective determinant for an anisotropic two-phase square checkerboard. Section 3 is devoted to a stability property in the general framework of periodic composites. In Section 4 we study the case of an anisotropic four-phase square checkerboard. Section 5 is devoted to the counter-example.

\section{Anisotropic two-phase checkerboards}

This Section is devoted to anisotropic two-phase square checkerboards. Under a low-contrast assumption between the two phases, the Tartar (1990) small-amplitude homogenisation formula allows us to write a second-order expansion of the effective coefficients. This expansion does not provide simple information on the whole homogenised matrix. Indeed, all the coefficients (that is 18 independent coefficients) of the expansions of the two phases appear in the final expansion of the effective matrix.

However, assuming that the conductivity matrices of the two phases commute, the expansion restricted to the effective determinant reduces to an explicit formula in the two phases. Moreover, it is remarkable that the zero-order term of this expansion gives the right effective determinant when the second-order term is zero.

\section{(a) Statement of the result}

- $Y:=\left(\frac{-1}{2}, \frac{1}{2}\right)^{2}$ is the unit square of $\mathbb{R}^{2}$; 
- $L_{\#}^{2}(Y)$ (resp. $\left.H_{\#}^{1}(Y)\right)$ is the set of the functions $\varphi$ in $L_{\text {loc }}^{2}\left(\mathbb{R}^{2}\right)\left(\right.$ resp. $\left.H_{\text {loc }}^{1}\left(\mathbb{R}^{2}\right)\right)$ and $Y$-periodic, i.e., $\varphi\left(y_{1}+1, y_{2}\right)=\varphi\left(y_{1}, y_{2}+1\right)=\varphi(y)$ a.e. $y \in \mathbb{R}^{2}$;

- for $\alpha, \beta>0, \mathcal{M}_{\# Y}(\alpha, \beta)$ is the set of the $Y$-periodic and symmetric matrixvalued functions $A$ such that, for all $\xi \in \mathbb{R}^{2}$,

$$
A(y) \xi \cdot \xi \geq \alpha|\xi|^{2} \text { and } A(y)^{-1} \xi \cdot \xi \geq \beta^{-1}|\xi|^{2} \quad \text { for a.e. } y \in Y \text {. }
$$

- for each $A \in \mathcal{M}_{\# Y}(\alpha, \beta)$, the homogenised matrix associated with the ma$\operatorname{trix} A$ is denoted by $A^{*}$ and is defined by the following formula (see e.g. Bensoussan et al. 1978), for all $\lambda \in \mathbb{R}^{2}$,

$$
A^{*} \lambda \cdot \lambda=\min \left\{\int_{Y} A(y)(\lambda+\nabla \varphi(y)) \cdot(\lambda+\nabla \varphi(y)) d y: \varphi \in H_{\#}^{1}(Y)\right\} .
$$

In this section we are interested by an anisotropic two-phase checkerboard structure. Let $A_{1}, A_{2}$ be two symmetric positive definite matrices of $\mathbb{R}^{2 \times 2}$ and let $A$ be the $Y$-periodic matrix-valued function defined by

$$
A:=(1-\chi) A_{1}+\chi A_{2}, \quad \text { where } \quad \chi:=1_{\left(0, \frac{1}{2}\right)^{2}}+1_{\left(-\frac{1}{2}, 0\right)^{2}} .
$$

In view of computing the determinant of the homogenised matrix of the two-phase checkerboard (2.3), we have the following result:

\section{Theorem 2.1.}

(i) Let $A_{0}$ be a symmetric positive definite matrix of $\mathbb{R}^{2 \times 2}$. Let $A_{1}, A_{2}$ be two symmetric positive definite matrices of $\mathbb{R}^{2 \times 2}$ such that $A_{1} A_{2}=A_{2} A_{1}$ and which admit the expansion $A_{j}=A_{0}+\delta B_{j}+\delta^{2} C_{j}+o\left(\delta^{2}\right), j=1,2$, around $A_{0}$. Then, the homogenised matrix $A^{*}$ of the checkerboard (2.3) satisfies, if $A_{0} \neq a_{0} I_{2}$,

$\operatorname{det} A^{*}-\sqrt{\operatorname{det}\left(A_{1} A_{2}\right)}=-F_{2}\left(A_{0}\right)\left(\operatorname{det} A_{1}-\operatorname{det} A_{2}\right)\left[\frac{\left(\operatorname{tr} A_{1}\right)^{2}}{\operatorname{det} A_{1}}-\frac{\left(\operatorname{tr} A_{2}\right)^{2}}{\operatorname{det} A_{2}}\right]+o\left(\delta^{2}\right)$.

If $A_{0}=a_{0} I_{2}, \operatorname{det} A^{*}-\sqrt{\operatorname{det}\left(A_{1} A_{2}\right)}=o\left(\delta^{2}\right)$. For any positive definite matrix $A \neq a I_{2}$

$$
F_{2}(A):=\frac{2}{\pi^{4}} \frac{1}{(a-b)^{2}+4 c^{2}} \sum_{n \in I^{2}} \frac{1}{(A n \cdot n)\left(A^{-1} n \cdot n\right)}\left[(a-b)^{2}+c^{2} \frac{\left(n_{1}^{2}-n_{2}^{2}\right)^{2}}{n_{1}^{2} n_{2}^{2}}\right],
$$

where $n=\left(n_{1}, n_{2}\right) \in I^{2}$ and where $I$ is the set of all odd integers.

(ii) The expansion (2.4) characterises exactly how the homogenised determinant differs from the simple formula $\sqrt{\operatorname{det}\left(A_{1} A_{2}\right)}$. From this perspective, it is optimal because for any symmetric positive definite matrices $A_{1}, A_{2}$ such that $A_{1} A_{2}=A_{2} A_{1}$, we have

$$
\left(\operatorname{det} A_{1}-\operatorname{det} A_{2}\right)\left[\frac{\left(\operatorname{tr} A_{1}\right)^{2}}{\operatorname{det} A_{1}}-\frac{\left(\operatorname{tr} A_{2}\right)^{2}}{\operatorname{det} A_{2}}\right]=0 \Longrightarrow \operatorname{det} A^{*}=\sqrt{\operatorname{det}\left(A_{1} A_{2}\right)} .
$$

Remark 2.2. Note that the term in factor of $F_{2}\left(A_{0}\right)$ in $(2.4)$ is a second-order term with respect to $\delta$. Part $(i i)$ provides an explicit formula of the effective determinant, which is apparently unrelated to the expansion introduced in part $(i)$. However, the positivity of $F_{2}\left(A_{0}\right)$ shows the converse implication of (2.6) holds true in expansion (2.4) up to higher order terms. 
(b) Proof of Theorem 2.1.

Proof of $(i)$. The proof is based on a small-amplitude homogenisation formula due to Tartar (1990). More precisely, Theorem 1.1, Example 2.1 and Theorem 4.2 in Tartar (1990) imply the following result:

Theorem 2.3 (Tartar). Let $A \in \mathcal{M}_{\# Y}(\alpha, \beta ; Y)$ be a $Y$-periodic matrix-valued function which admits the following second-order expansion around the symmetric positive definite matrix $A_{0}$ :

$$
A=A_{0}+\delta B+\delta^{2} C+o\left(\delta^{2}\right), \quad \text { where } B, C \text { are } Y \text {-periodic. }
$$

Then, the homogenised matrix $A^{*}$ defined by (2.2) satisfies

$$
A^{*}=A_{0}+\delta B_{0}+\delta^{2}\left(C_{0}-M\right)+o\left(\delta^{2}\right), \quad \text { where } \quad B_{0}:=\int_{Y} B, \quad C_{0}:=\int_{Y} C
$$

and the correction matrix $M$ is defined by

$$
M_{i j}:=\sum_{k, l}^{2} \sum_{n \in \mathbb{Z}^{2} \backslash\{0\}} B_{i k}^{n} \overline{B_{j l}^{n}} \frac{n_{k} n_{l}}{A_{0} n \cdot n} \quad \text { with } \quad B^{n}:=\int_{Y} B(y) e^{-2 i \pi n \cdot y} d y .
$$

In the present case, the matrix-valued functions $A, B, C$ of (2.7) have the twophase checkerboard structure (2.3) and the corresponding two-phases satisfy the second-order expansion $A=A_{0}+\delta B_{j}+\delta^{2} C_{j}+o\left(\delta^{2}\right)$ for $j=1,2$. The Fourier coefficients of $B$ (with the two-phases $B_{1}, B_{2}$ ) are given by

$$
B^{n}=\frac{2}{\pi^{2} n_{1} n_{2}}\left(B_{1}-B_{2}\right) \text { if } n_{1}, n_{2} \in I \text { (odd integers) and } B^{n}=0 \text { otherwise. }
$$

Denote $A_{0}:=\left(\begin{array}{ll}a_{0} & c_{0} \\ c_{0} & b_{0}\end{array}\right)$ and $B_{j}:=\left(\begin{array}{cc}\alpha_{j} & \gamma_{j} \\ \gamma_{j} & \beta_{j}\end{array}\right)$ for $j=1,2$. Then, putting the value of $B^{n}$ in formula (2.9) yields the coefficients $M_{11}, M_{22}$ and $M_{12}$ of the correction matrix $M$ :

$$
\left\{\begin{aligned}
M_{11}= & S_{1}\left(\alpha_{1}-\alpha_{2}\right)^{2}+S_{2}\left(\gamma_{1}-\gamma_{2}\right)^{2}+T\left(\alpha_{1}-\alpha_{2}\right)\left(\gamma_{1}-\gamma_{2}\right) \\
M_{22}= & S_{1}\left(\gamma_{1}-\gamma_{2}\right)^{2}+S_{2}\left(\beta_{1}-\beta_{2}\right)^{2}+T\left(\beta_{1}-\beta_{2}\right)\left(\gamma_{1}-\gamma_{2}\right) \\
M_{12}= & S_{1}\left(\alpha_{1}-\alpha_{2}\right)\left(\gamma_{1}-\gamma_{2}\right)+S_{2}\left(\beta_{1}-\beta_{2}\right)\left(\gamma_{1}-\gamma_{2}\right) \\
& +\frac{T}{2}\left[\left(\alpha_{1}-\alpha_{2}\right)\left(\beta_{1}-\beta_{2}\right)+\left(\gamma_{1}-\gamma_{2}\right)^{2}\right]
\end{aligned}\right.
$$

where $S_{1}, S_{2}, T$ are the series

$$
\left\{\begin{array}{l}
S_{1}:=\frac{4}{\pi^{4}} \sum_{n \in I^{2}} \frac{1}{n_{2}^{2}} \frac{1}{A_{0} n \cdot n}, \quad S_{2}:=\frac{4}{\pi^{4}} \sum_{n \in I^{2}} \frac{1}{n_{1}^{2}} \frac{1}{A_{0} n \cdot n} \\
T:=\frac{8}{\pi^{4}} \sum_{n \in I^{2}} \frac{1}{n_{1}^{2} n_{2}^{2}} \frac{1}{A_{0} n \cdot n} .
\end{array}\right.
$$

Taking into account the equalities $A_{1} A_{2}=A_{2} A_{1}$ and $a_{0} S_{1}+b_{0} S_{2}+c_{0} T=1 / 4$, we can compute by means of Maple the second-order expansion of $\operatorname{det} A^{*}$ from formulae (2.8) and (2.10) which reads as $\operatorname{det} A^{*}=\sqrt{\operatorname{det}\left(A_{1} A_{2}\right)}+\delta^{2} X+o\left(\delta^{2}\right)$, 
where $X$ is given by a very long formula depending on the coefficients of $A_{0}, B_{j}, C_{j}$ for $j=1,2$. Alternatively, we also have

$$
\left(\operatorname{det} A_{1}-\operatorname{det} A_{2}\right)\left[\frac{\left(\operatorname{tr} A_{1}\right)^{2}}{\operatorname{det} A_{1}}-\frac{\left(\operatorname{tr} A_{2}\right)^{2}}{\operatorname{det} A_{2}}\right]=\delta^{2} Y+o\left(\delta^{2}\right) .
$$

Factorising the terms $X$ and $Y$ with Maple yields $X=-F_{2}\left(A_{0}\right) Y$ if $A_{0} \neq a_{0} I_{2}$ and $X=0$ otherwise. A lengthy but straightforward computation shows that $F_{2}$ can be written in a simple form, formula (2.5). This concludes the proof of expansion (2.4). Proof of $(\mathrm{ii})$. The proof is based upon a duality argument introduced by Keller (1964). Let $\tilde{A}:=(1-\chi) A_{2}+\chi A_{1}$ be the checkerboard structure obtained by exchanging the phases $A_{1}$ and $A_{2}$. The matrix $\tilde{A}$ corresponds to the same structure as that of $A$ up to a translation of vector $(0,1 / 2)$, and thus yields the same homogenised matrix $A^{*}$. We will now find the best constant $k>0$ such that $\tilde{A} \geq k \frac{A}{\operatorname{det} A}$ a.e. in $Y$. Let $\lambda_{j}$ and $\mu_{j}$ be the eigenvalues of $A_{j}$ for $j=1,2$, with respect to the same basis of eigenvectors. We may then write

$$
\begin{array}{cc}
A_{1} \geq k \frac{A_{2}}{\operatorname{det} A_{2}} \Leftrightarrow \lambda_{1} \geq k \frac{\lambda_{2}}{\lambda_{2} \mu_{2}} \text { and } \mu_{1} \geq k \frac{\mu_{2}}{\lambda_{2} \mu_{2}} & \Leftrightarrow k \leq \min \left(\lambda_{1} \mu_{2}, \lambda_{2} \mu_{1}\right) \\
\text { by symmetry } & \Leftrightarrow A_{2} \geq k \frac{A_{1}}{\operatorname{det} A_{1}}
\end{array}
$$

The best choice is thus $k:=\min \left(\lambda_{1} \mu_{2}, \lambda_{2} \mu_{1}\right)$, which in turns implies $\tilde{A} \geq k \frac{A}{\operatorname{det} A}$ a.e. in $Y$. Thanks to a result due to Mendelson 1975 (see also Nevard \& Keller 1985 and Francfort \& Murat 1987), we have

$$
\left(\frac{A}{\operatorname{det} A}\right)^{*}=\frac{A^{*}}{\operatorname{det} A^{*}}
$$

Moreover, definition (2.2) implies that $B \leq C \Rightarrow B^{*} \leq C^{*}$. We thus deduce

$$
\tilde{A}^{*}=A^{*} \geq k\left(\frac{A}{\operatorname{det} A}\right)^{*}=k \frac{A^{*}}{\operatorname{det} A^{*}}
$$

which implies $\operatorname{det} A^{*} \geq k$, i.e., $\operatorname{det} A^{*} \geq \min \left(\lambda_{1} \mu_{2}, \lambda_{2} \mu_{1}\right)$. Replacing $A$ by $\frac{A}{\operatorname{det} A}$ also yields

$$
\operatorname{det}\left(\frac{A^{*}}{\operatorname{det} A^{*}}\right)=\frac{1}{\operatorname{det} A^{*}} \geq \min \left(\frac{1}{\mu_{1}} \frac{1}{\lambda_{2}}, \frac{1}{\mu_{2}} \frac{1}{\lambda_{1}}\right)=\frac{1}{\max \left(\lambda_{1} \mu_{2}, \lambda_{2} \mu_{1}\right)} .
$$

Therefore, we obtain

$$
\min \left(\lambda_{1} \mu_{2}, \lambda_{2} \mu_{1}\right) \leq \operatorname{det} A^{*} \leq \max \left(\lambda_{1} \mu_{2}, \lambda_{2} \mu_{1}\right) .
$$

On the other hand, we have

$$
\frac{\left(\operatorname{tr} A_{1}\right)^{2}}{\operatorname{det} A_{1}}=\frac{\left(\operatorname{tr} A_{2}\right)^{2}}{\operatorname{det} A_{2}} \Leftrightarrow \frac{\lambda_{1}}{\mu_{1}}+\frac{\mu_{1}}{\lambda_{1}}=\frac{\lambda_{2}}{\mu_{2}}+\frac{\mu_{2}}{\lambda_{2}} \Leftrightarrow \lambda_{1} \mu_{2}=\lambda_{2} \mu_{1} \text { or } \lambda_{1} \lambda_{2}=\mu_{1} \mu_{2} .
$$

Let us conclude:

- If $\lambda_{1} \mu_{2}=\lambda_{2} \mu_{1}$ then (2.13) implies that $\operatorname{det} A^{*}=\lambda_{1} \mu_{2}=\sqrt{\operatorname{det}\left(A_{1} A_{2}\right)}$.

- If $\lambda_{1} \lambda_{2}=\mu_{1} \mu_{2}$ then $A_{1} A_{2}=\lambda_{1} \lambda_{2} I_{2}$. Whence by a result of Dykhne (1970), $A^{*}=\sqrt{\lambda_{1} \lambda_{2}} I_{2}$ and we still obtain $\operatorname{det} A^{*}=\sqrt{\operatorname{det}\left(A_{1} A_{2}\right)}$.

- If $\operatorname{det} A_{1}=\operatorname{det} A_{2}$ then again by Dykhne, $\operatorname{det} A^{*}=\operatorname{det} A_{1}=\sqrt{\operatorname{det}\left(A_{1} A_{2}\right)}$. 


\section{Computation of the homogenised determinant of some microstructures}

In this section we introduce a stability principle in order to compute the effective determinant for some periodic composites. To this end, we study the effects of axial distortions (with respect to the $x_{1}$ and $x_{2}$ axes) on the effective properties of a given composite. The stability property then means that the effective determinant of the modified composite (under an axial distortion) reads as the product of the distortion by the effective determinant of the initial composite, without any other interaction.

For example, laminated composites (whose conductivity depends on one direction) and isotropic four-phase rectangular checkerboards (studied in Craster \& Obnosov 2001) satisfy the stability property. More generally, for any periodic composite satisfying the stability property we obtain an explicit formula for the effective determinant. In fact, two formulae are derived corresponding to the two axial distortions. This approach by stability will also allow us to construct an explicit second-order expansion for anisotropic four-phase square checkerboards in Section 4.

(a) A stability under axial deformation property

In the sequel, $\Omega$ is a bounded open subset of $\mathbb{R}^{d}, d \geq 1$, and $\mathcal{M}_{\Omega}(\alpha, \beta)$, for $\alpha, \beta>0$, is the set of the symmetric invertible matrix-valued functions $A$ which satisfy (2.1) on $\Omega$. We shall make use of the theoretical approach to homogenisation introduced by Murat \& Tartar $(1978,1997)$, the $H$-convergence.

Definition 3.1 (Murat-Tartar). A sequence $A^{\varepsilon}$ of $\mathcal{M}_{\Omega}(\alpha, \beta)$ is said to $H$-converge to a matrix-valued $A^{*}$ if for any $f$ in $H^{-1}(\Omega)$, the solution $u^{\varepsilon}$ in $H_{0}^{1}(\Omega)$ of $\operatorname{div}\left(A^{\varepsilon} \nabla u^{\varepsilon}\right)=$ $f$ in $\mathcal{D}^{\prime}(\Omega)$, satisfies the weak convergences

$$
u^{\varepsilon} \rightarrow u^{*} \text { in } H_{0}^{1}(\Omega) \quad \text { and } \quad A^{\varepsilon} \nabla u^{\varepsilon} \rightarrow A^{*} \nabla u^{*} \text { in } L^{2}(\Omega)^{d},
$$

where $u^{*}$ is the solution of $\operatorname{div}\left(A^{*} \nabla u^{*}\right)=f$ in $\mathcal{D}^{\prime}(\Omega)$.

The matrix-valued $A^{*}$ in 3.1 is called the $H$-limit of $A^{*}$ and also belongs to the set $\mathcal{M}_{\Omega}(\alpha, \beta)$. We shall always assume that $A^{\varepsilon}$ is a sequence of $\mathcal{M}_{\Omega}(\alpha, \beta)$. We shall also use the following notation convention: the $H$-limit of a positive definite sequence of matrices $A^{\varepsilon}$ is noted $A^{*}$, and $A_{B}^{*}$ corresponds to the $H$-limit of the sequence $B A^{\varepsilon} B$.

Definition 3.2. Let $A^{\varepsilon}$ be a sequence of positive definite matrix-valued functions, which $H$-converges to $A^{*}$. The limit microstructure corresponding to $A^{*}$ is said to be stable under deformation if for any constant symmetric positive definite matrix $B$, we have

$$
\operatorname{det} A_{B}^{*}=(\operatorname{det} B)^{2} \operatorname{det} A^{*} .
$$

It is said to be stable under axial deformation if the property holds for any constant diagonal positive definite matrix $B$.

Note that $A_{B}^{*}$ is defined a priori up to the extraction of a subsequence. In that way, $A_{B}^{*}$ could correspond to subsequence dependent $H$-limits. In such a case, the definition of stability under deformation is that (3.1) stands for all subsequences. 
The choice of the word deformation in this definition is explained by the following proposition:

Proposition 3.3. For any sequence of positive definite matrix-valued functions $A^{\varepsilon}$ converging to an $H$-limit $A^{*}$ and any constant positive definite matrix $B$, we have $B\left[A^{\varepsilon}(B \cdot)\right]^{*} B=A_{B}^{*}(B \cdot)$ a.e. in $B^{-1} \Omega$, where $\left[A^{\varepsilon}(B \cdot)\right]^{*}$ is the H-limit of the sequence $x \mapsto A^{\varepsilon}(B x)$. So, a limit microstructure with constant $H$-limit is stable under deformation if and only if

$$
\operatorname{det}\left[A^{\varepsilon}(B \cdot)\right]^{*}=\operatorname{det} A^{*} .
$$

Proof. Let us note $A_{1}$ the $H$-limit $\left[A^{\varepsilon}(B \cdot)\right]^{*}$. Consider the Dirichlet problem

$$
-\operatorname{div}\left(A^{\varepsilon}(B x) \nabla u^{\varepsilon}\right)=f \text { in } B^{-1} \Omega, \quad u^{\varepsilon}=0 \text { on } \partial\left(B^{-1} \Omega\right),
$$

for some $f \in H^{-1}\left(B^{-1} \Omega\right)$. After an integration by parts against a test function $\phi$ we obtain

$$
\int_{B^{-1} \Omega} A^{\varepsilon}(B x) \nabla u^{\varepsilon} \cdot \nabla \phi d x=\int_{B^{-1} \Omega} f \phi d x
$$

which is also, after the change of variable $y=B x$, and the notations $\psi(y):=\phi(x)$, $v^{\varepsilon}(y):=u^{\varepsilon}(x)$,

$$
\int_{\Omega} B A^{\varepsilon}(y) B \nabla v^{\varepsilon} \cdot \nabla \psi \operatorname{det} B^{-1} d y=\int_{\Omega} g \psi \operatorname{det} B^{-1} d y
$$

Passing to the limit as $\varepsilon$ tends to 0 in (3.3) we obtain

$$
\int_{B^{-1} \Omega} A_{1}(x) \nabla u^{*} \cdot \nabla \phi d x=\int_{B^{-1} \Omega} f \phi d y,
$$

where $u^{*}$ is the weak limit of $u^{\varepsilon}$ in $H_{0}^{1}\left(B^{-1} \Omega\right)$. Alternatively, passing to the limit in (3.4) we obtain

$$
\begin{aligned}
\int_{\Omega} A_{B}^{*}(y) \nabla v^{\varepsilon} \cdot \nabla \psi \operatorname{det} B^{-1} d y & =\int_{\Omega} g \psi \operatorname{det} B^{-1} d y \\
\int_{B^{-1} \Omega} B^{-1} A_{B}^{*}(B x) B^{-1} \nabla u^{*} \cdot \nabla \phi d x & =\int_{B^{-1} \Omega} f \phi d x .
\end{aligned}
$$

By the uniqueness of the limit problem, we have $A_{1}=B^{-1} A_{B}^{*}(B \cdot) B^{-1}$.

This result is known in the general case where $B=\nabla \varphi$ with $\varphi$ a diffeomorphism (see e.g. Tartar 2000). The following proposition gives examples of microstructures which are stable under (axial) deformation:

\section{Proposition 3.4.}

(a) Any isotropic laminated microstructure is stable under deformation, in any dimension.

(b) More generally, multipliable microstructures (in the sense of Fabre \& Mossino 1998) are stable under deformation.

(c) In two dimension any isotropic four-phase checkerboard is stable under axial deformation. 
In the sequel, unless otherwise specified, we will simply write that a matrix or its corresponding microstructure is stable to indicate that it is stable under axial deformation.

Proof. (a) For $x \in \mathbb{R}^{n}$, let $A^{\varepsilon}(x)=A^{\varepsilon}(\xi \cdot x)$ be a symmetric matrix, with $|\xi|=1$. It is well known (the original proof being from Murat \& Tartar 1978, 1997) that up to the extraction of a subsequence, the homogenised matrix $A^{*}$ is given by

$$
A^{*} \xi \cdot \xi=\left(\lim \frac{1}{A^{\varepsilon} \xi \cdot \xi}\right)^{-1}, \quad A^{*} \xi=A^{*} \xi \cdot \xi \lim \left(\frac{A^{\varepsilon} \xi}{A^{\varepsilon} \xi \cdot \xi}\right) \text { in } L^{\infty}(\Omega) \text { weak-*, }
$$

and, for all $\lambda$ such that $\lambda \cdot \xi=0$,

$$
\left(A^{*}-\frac{A^{*} \xi \otimes A^{*} \xi}{A^{*} \xi \cdot \xi}\right) \lambda=\lim \left(A^{\varepsilon}-\frac{A^{\varepsilon} \xi \otimes A^{\varepsilon} \xi}{A^{\varepsilon} \xi \cdot \xi}\right) \lambda \quad \text { in } L^{\infty}(\Omega) \text { weak- * . }
$$

If $A^{\varepsilon}$ is an isotropic matrix, $A^{\varepsilon}=a^{\varepsilon}(\xi \cdot x) I_{n}$, with $a^{\varepsilon} \in L^{\infty}(\mathbb{R})$, and $B$ be a positive definite symmetric matrix, the above formulae simplifies into $A_{B}^{*} \xi \cdot \xi=\underline{a}|\eta|^{2}$, $A_{B}^{*} \xi=\underline{a} B \eta$, and

$$
\begin{gathered}
A_{B}^{*} \lambda-\underline{a} \frac{B \eta \otimes B \eta}{\eta \cdot \eta} \lambda=\bar{a}\left(B^{2}-\frac{B \eta \otimes B \eta}{\eta \cdot \eta}\right) \lambda, \\
\text { which implies } A_{B}^{*}=B\left(\bar{a} I_{n}-(\bar{a}-\underline{a}) \frac{\eta \otimes \eta}{\eta \cdot \eta}\right) B,
\end{gathered}
$$

with $\eta:=B \xi$, and where $\underline{a}$ (resp. $\bar{a}$ ) is the harmonic (resp. arithmetic) average of $a^{\varepsilon}$. We thus obtain $\operatorname{det} A_{B}^{*}=(\operatorname{det} B)^{2}(\bar{a})^{n-1} \underline{a}$, which proves the stability by deformation.

(b) For a multipliable microstructure $A^{\varepsilon}$, that is, for which there exists $M^{\varepsilon}$ and $P^{\varepsilon}$ such that:

- $M^{\varepsilon} A^{\varepsilon}=P^{\varepsilon}$ with $\left(M^{\varepsilon}, P^{\varepsilon}\right) \neq(0,0), M^{\varepsilon}=\left[m_{i j}^{\varepsilon}\left(x_{j}\right)\right]_{i j}, P^{\varepsilon}=\left[p_{i j}^{\varepsilon}\left(x_{j}^{\prime}\right)\right]_{i j}$ where $x \in \Omega$ is denoted by $x=\left(x_{j}, x_{j}^{\prime}\right), x_{j} \in \mathbb{R}$,

- $M^{\varepsilon}$ and $P^{\varepsilon}$ converge respectively to $M$ and $P$ in $L^{\infty}(\Omega)$ weak-*,

- $M$ is invertible.

Fabre and Mossino then showed that $A^{*}=M^{-1} P$. Note that, for a constant positive definite matrix $B$, the microstructure $A_{B}^{\varepsilon}$ is also multipliable since the matrices $M_{B}^{\varepsilon}:=M^{\varepsilon} B^{-1}$ and $P_{B}^{\varepsilon}:=P^{\varepsilon} B$ satisfy the requirements. Clearly, $M_{B}^{\varepsilon}$ converges to $M_{B}:=M B^{-1}$ and $P_{B}^{\varepsilon}$ converges to $P_{B}:=P B$. Invoking again the Fabre-Mossino result, $A_{B}^{*}=M_{B}^{-1} P_{B}=B A^{*} B$, and in particular, $A$ is stable under deformation.

(c) Let us now turn to the case of an isotropic two-dimensional four-phase periodic checkerboard, that is, with $A$ defined by

$$
A(x):=\left\{\begin{array}{llll}
a I_{2} & \text { for } x \in\left(-\frac{1}{2}, 0\right) \times\left(0, \frac{1}{2}\right) & b I_{2} & \text { for } x \in\left(0, \frac{1}{2}\right) \times\left(0, \frac{1}{2}\right) \\
c I_{2} & \text { for } x \in\left(0, \frac{1}{2}\right) \times\left(-\frac{1}{2}, 0\right) & d I_{2} & \text { for } x \in\left(-\frac{1}{2}, 0\right) \times\left(-\frac{1}{2}, 0\right)
\end{array}\right.
$$

and repeated periodically. Note that, since it is a periodic structure, the $H$-limit is constant, and by (3.2) the invariance by axial deformation amounts to $\operatorname{det} A^{*}=$ $\operatorname{det}[A(B \cdot)]^{*}$. For any positive definite diagonal matrix $B=\operatorname{diag}\left(b_{1}, b_{2}\right)$, the microstructure corresponding to $A(B \cdot)$ corresponds to a four-phase (of equal area) 
rectangular $b_{1}^{-1} \times b_{2}^{-1}$ checkerboard. In both cases, the homogenised matrices $A^{*}$ and $[A(B \cdot)]^{*}$ have been obtained by Craster \& Obnosov (2001, p. 8), and their common determinant is

$$
\operatorname{det} A^{*}=\operatorname{det}[A(B \cdot)]^{*}=\frac{b c d+a c d+a b d+a b c}{a+b+c+d} .
$$

(b) Computation of the homogenised determinant for a stable two-dimensional microstructure

In order to state this result, we introduce the following notation: for an integrable and $Y$-periodic function $f$ of one or two variables, we note $m_{i}(f)$ the arithmetic average of $f$ with respect to the $i$ th variable:

$$
m_{1}(f)(\cdot):=\int_{-\frac{1}{2}}^{\frac{1}{2}} f\left(x_{1}, \cdot\right) d x_{1} \quad \text { and } \quad m_{2}(f)(\cdot):=\int_{-\frac{1}{2}}^{\frac{1}{2}} f\left(\cdot, x_{2}\right) d x_{2} .
$$

Theorem 3.5. Let $A^{\varepsilon}(x):=A(x / \varepsilon)$ be a stable $\varepsilon Y$-periodic microstructure. Then, the corresponding effective determinant is given by the two following formulae

$$
\begin{aligned}
& \operatorname{det} A^{*}=\left.\frac{m_{2}\left(\frac{A_{11} A_{22}-A_{12} A_{21}}{A_{11}}\right)}{m_{1}\left(\frac{A_{12}}{A_{11}}\right) m_{1}\left(\frac{A_{21}}{A_{11}}\right)+m_{1}\left(\frac{1}{A_{11}}\right) m_{1}\left(\frac{A_{11} A_{22}-A_{12} A_{21}}{A_{11}}\right)}\right) \\
& m_{1}\left(\frac{1}{A_{11}}\right)\left.\frac{m_{2}\left(\frac{m_{2}\left(\frac{A_{11} A_{22}-A_{12} A_{21}}{A_{22}}\right)}{m_{1}\left(\frac{A_{12}}{A_{11}}\right) m_{1}\left(\frac{A_{21}}{A_{11}}\right)+m_{1}\left(\frac{1}{A_{11}}\right) m_{22}-A_{12} A_{21}}\right)}{A_{11}}\right) \\
& \operatorname{det} A^{*}=\left.\frac{m_{1}\left(\frac{1}{m_{2}\left(\frac{A_{12}}{A_{22}}\right) m_{2}\left(\frac{A_{21}}{A_{22}}\right)+m_{2}\left(\frac{1}{A_{22}}\right) m_{2}\left(\frac{A_{11} A_{22}-A_{12} A_{21}}{A_{22}}\right)}\right)}{m_{2}\left(\frac{1}{A_{22}}\right)}\right) \\
& m_{1}\left(\frac{m_{2}\left(\frac{1}{A_{22}}\right) m_{2}\left(\frac{A_{11} A_{22}-A_{12} A_{21}}{A_{22}}\right)}{m_{2}\left(\frac{A_{12}}{A_{22}}\right) m_{2}\left(\frac{A_{21}}{A_{22}}\right)+m_{2}}\right)
\end{aligned}
$$

Remark 3.6. If $A$ is diagonal, formulae (3.5) and (3.6) simplify into

$$
\operatorname{det} A^{*}=\frac{m_{2}\left(\left[m_{1}\left(A_{11}^{-1}\right)\right]^{-1}\right)}{m_{2}\left(\left[m_{1}\left(A_{22}\right)\right]^{-1}\right)} \quad \text { and } \quad \operatorname{det} A^{*}=\frac{m_{1}\left(\left[m_{2}\left(A_{22}^{-1}\right)\right]^{-1}\right)}{m_{1}\left(\left[m_{2}\left(A_{11}\right)\right]^{-1}\right)}
$$

Proof of Theorem 3.5. By a rotation of angle $\pi / 2$ of the periodic pattern, formula (3.5) yields formula (3.6). We will thus prove (3.5). Let $A(y)$ be a $Y$-periodic microstructure. For $p, q \in \mathbb{N}^{2}$, let $A_{p, q}$ be defined as $A_{p, q}(y):=A\left(p y_{1}, q y_{2}\right)$. Note that $A_{p, q}=A(B \cdot)$ where $B$ is the diagonal positive definite matrix with entries $p$ and $q$. By hypothesis $A$ is stable, therefore $\operatorname{det} A^{*}=\operatorname{det} A_{p, q}^{*}$ for all positive $p, q$. 
Passing to the limit in $p$ and in $q$, we will obtain (3.5). We first assume that $q$ is fixed. Since $A$ and in turn $A_{p, q}$ are $Y$-periodic, the homogenised matrix $A_{p, q}^{*}$ is given in terms of its correctors $\chi_{p}^{1}, \chi_{p}^{2}$ by

$$
\forall \lambda \in \mathbb{R}^{2}, \quad A_{p, q}^{*} \lambda=\int_{Y} A_{p, q}(y)\left[\lambda+\nabla \chi_{p}^{\lambda}(y)\right] d y,
$$

with the notation $\chi_{p}^{\lambda}:=\sum_{k=1}^{2} \chi_{p}^{k} \lambda_{k}$ and where $\chi_{p}^{\lambda}$ is the unique solution in $H_{\#}^{1}(Y) / \mathbb{R}$ of the cell problem

$$
\operatorname{div}\left(A_{p, q}(y)\left[\lambda+\nabla \chi_{p}^{\lambda}\right]\right)=0 \quad \text { in } \mathcal{D}^{\prime}\left(\mathbb{R}^{2}\right) .
$$

Note that the sequence $\chi_{p}^{\lambda}$ is bounded in $H_{\#}^{1}(Y)$ uniformly in $p$, and therefore up to a subsequence, converges weakly to a limit $\chi^{\lambda}$. Hence (see Allaire 1992) there exists a function $X^{\lambda}(y, z)$ in $L_{\#}^{2}\left(Y, H_{\#}^{1}(Y) / \mathbb{R}\right)$ such that, up to a subsequence, $\nabla \chi_{p}^{\lambda}$ two-scale converges to $\nabla \chi^{\lambda}(y)+\nabla_{z} X^{\lambda}(y, z)$. Note that the matrices $A_{p, q}$ converges strongly in the sense of two-scale convergence towards $A\left(z_{1}, y_{2}\right)$, that is

$$
\lim _{p \rightarrow+\infty} \int_{Y}\left(A_{p, q}(y)\right)^{2} d y=\int_{Y} \int_{Y}\left(A\left(z_{1}, y_{2}\right)\right)^{2} d y d z .
$$

So, for any $\phi \in C_{\#}^{\infty}(Y)$ and $\phi_{1} \in C_{\#}^{\infty}(Y \times Y), A\left(p y_{1}, q y_{2}\right)\left[\nabla \phi(y)+\nabla_{z} \phi_{1}(y, p y)\right]$ is an admissible test function, that converges strongly in the sense of two-scale convergence to its two-scale limit. Multiplying (3.8) by $\phi(y)+p^{-1} \phi_{1}(y, p y)$ and integrating by parts, we obtain

$$
\int_{Y} A\left(p y_{1}, q y_{2}\right)\left[\lambda+\nabla \chi_{p}^{\lambda}(y)\right] \cdot\left[\nabla \phi(y)+\nabla_{z} \phi_{1}(y, p y)+p^{-1} \nabla_{y} \phi_{1}(y, p y)\right] d y=0 .
$$

Passing to the limit as $p$ tends to $+\infty$, we obtain

$$
\iint_{Y^{2}} A\left(z_{1}, q y_{2}\right)\left[\lambda+\nabla \chi^{\lambda}(y)+\nabla_{z} X^{\lambda}(y, z)\right] \cdot\left[\nabla \phi(y)+\nabla_{z} \phi_{1}(y, z)\right] d y d z=0,
$$

and by $(3.7)$

$$
\lim _{p \rightarrow+\infty} A_{p, q}^{*} \lambda=\int_{Y} \int_{Y} A\left(z_{1}, q y_{2}\right)\left[\lambda+\nabla \chi^{\lambda}(y)+\nabla_{z} X^{\lambda}(y, z)\right] d y d z .
$$

By density, equation (3.9) holds true for all $\left(\phi, \phi_{1}\right) \in H_{\#}^{1}(Y) \times L_{\#}^{2}\left(Y, H_{\#}^{1}(Y) / \mathbb{R}\right)$. Computing the corrector $X^{\lambda}$ which is the classic corrector for laminates in the $y_{1}$ direction, we obtain that $\chi^{\lambda}$ is solution of $\operatorname{div}\left(A_{\infty}\left(q y_{2}\right)\left[\lambda+\nabla \chi^{\lambda}(y)\right]\right)=0$ in $\mathcal{D}^{\prime}\left(\mathbb{R}^{2}\right)$ and that (3.10) simplifies into

$$
\lim _{p \rightarrow+\infty} A_{p, q}^{*} \lambda=\int_{Y} A_{\infty}\left(q y_{2}\right)\left[\lambda+\nabla \chi^{\lambda}(y)\right] d y
$$

where $A_{\infty}$ is the function of the second variable given by $\left[A_{\infty}\right]_{11}=\left(m_{1}\left(1 / A_{11}\right)\right)^{-1}$, $\left[A_{\infty}\right]_{21}=\left[A_{\infty}\right]_{11} m_{1}\left(A_{21} / A_{11}\right),\left[A_{\infty}\right]_{12}=\left[A_{\infty}\right]_{11} m_{1}\left(A_{12} / A_{11}\right)$, and $\left[A_{\infty}\right]_{22}=$ $\left[A_{\infty}\right]_{11} m_{1}\left(A_{12} / A_{11}\right) m_{1}\left(A_{21} / A_{11}\right)+m_{1}\left(\left(A_{11} A_{22}-A_{12} A_{21}\right) / A_{11}\right)$. Note that

$$
\operatorname{det} A_{\infty}=\left(m_{1}\left(\frac{1}{A_{11}}\right)\right)^{-1} m_{1}\left(\frac{A_{11} A_{22}-A_{12} A_{21}}{A_{11}}\right) .
$$




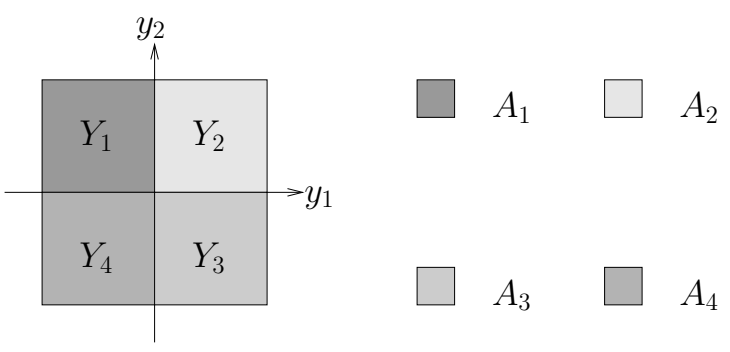

Figure 1. A four-phase checkerboard.

We now consider $A_{\infty}(q \cdot)$ as an oscillating sequence of period $q^{-1}$. The same arguments applies and we can again pass to the limit in $q$ in formula (3.11). A new homogenised matrix appears $A_{\infty, \infty}$ which is constant. As a consequence,

$$
\lim _{q \rightarrow+\infty}\left(\lim _{p \rightarrow+\infty} A_{p, q}^{*} \lambda\right)=\int_{Y} A_{\infty, \infty} d y=A_{\infty, \infty} .
$$

The determinant of $A_{\infty, \infty}$ can be obtained from (3.12), exchanging the roles of the first and second indexes, and substituting $A_{\infty}$ for $A$. This gives

$$
\operatorname{det} A_{\infty, \infty}=\left(m_{2}\left(1 /\left[A_{\infty}\right]_{22}\right)\right)^{-1} m_{2}\left(\left(\left[A_{\infty}\right]_{11}\left[A_{\infty}\right]_{22}-\left[A_{\infty}\right]_{12}\left[A_{\infty}\right]_{21}\right) /\left[A_{\infty}\right]_{22}\right),
$$

which is $(3.5)$.

Remark 3.7. For a diagonal periodic matrix $A$, taking the test function $\varphi$ depending of the first (or second) variable only in the homogenised formula (2.2) yields,

$$
A_{11}^{*} \leq\left(m_{1}\left(\left[m_{2}\left(A_{11}\right)\right]^{-1}\right)\right)^{-1} \text { and } A_{22}^{*} \leq\left(m_{2}\left(\left[m_{1}\left(A_{22}\right)\right]^{-1}\right)\right)^{-1} .
$$

Using (as in the proof of Theorem 2.1) the identity (2.12) we also obtain

$$
\left(\operatorname{det} A^{*}\right) / A_{11}^{*} \geq m_{1}\left(\left[m_{2}\left(A_{22}^{-1}\right)\right]^{-1}\right) \text { and }\left(\operatorname{det} A^{*}\right) / A_{22}^{*} \geq m_{2}\left(\left[m_{1}\left(A_{11}^{-1}\right)\right]^{-1}\right) .
$$

Formulae (3.6) are the products of these upper and lower bounds. In the special case when the microstructure $A$ is diagonal with separable variables, i.e., $A_{j j}\left(x_{1}, x_{2}\right)=$ $a_{j j}\left(x_{1}\right) b_{j j}\left(x_{2}\right), j=1,2$, the four inequalities above are equalities (in such a case, the structure is multipliable, as it is explained in Proposition 3.4).

\section{Checkerboard with four anisotropic phases}

In the previous Section, we noticed that any isotropic four-phase checkerboard is stable in the sense of 3.2. It is then natural to ask if anisotropic checkerboards are stable. We restrict ourselves to a checkerboard with four diagonal phases $A_{j}=$ $\operatorname{diag}\left(a_{j}, b_{j}\right), j=1, \ldots, 4$ (see Figure 1 on page 12 ), whose periodic matrix is defined in the unit square $Y$ by

$$
A:=1_{Y_{1}} A_{1}+1_{Y_{2}} A_{2}+1_{Y_{3}} A_{3}+1_{Y_{4}} A_{4},
$$

where $Y_{1}:=(-1 / 2,0) \times(0,1 / 2), Y_{2}:=(0,1 / 2) \times(0,1 / 2), Y_{3}:=(0,1 / 2) \times(-1 / 2,0)$ and $Y_{4}:=(-1 / 2,0) \times(-1 / 2,0)$. We still denote by $A^{*}$ its homogenised matrix. 
Omitting to verify that the microstructure $A$ is stable under axial deformation, we can compute the values of the homogenised determinants $D^{*}$ and $\tilde{D}^{*}$ given by (3.6). We obtain

$$
\begin{aligned}
& D^{*}=\frac{a_{2} a_{3} a_{4}+a_{1} a_{3} a_{4}+a_{1} a_{2} a_{4}+a_{1} a_{2} a_{3}}{\left(a_{1}+a_{2}\right)\left(a_{3}+a_{4}\right)} \times \frac{\left(b_{1}+b_{2}\right)\left(b_{3}+b_{4}\right)}{b_{1}+b_{2}+b_{3}+b_{4}}, \\
& \text { and } \tilde{D}^{*}=\frac{b_{2} b_{3} b_{4}+b_{1} b_{3} b_{4}+b_{1} b_{2} b_{4}+b_{1} b_{2} b_{3}}{\left(b_{1}+b_{4}\right)\left(b_{2}+b_{3}\right)} \times \frac{\left(a_{1}+a_{4}\right)\left(a_{2}+a_{3}\right)}{a_{1}+a_{2}+a_{3}+a_{4}}
\end{aligned}
$$

Both formulae are equal if $E(a)=E(b)$ with $a:=\left(a_{1}, \ldots, a_{4}\right), b:=\left(b_{1}, \ldots, b_{4}\right)$ and

$$
E(a):=\frac{\left(a_{2} a_{3} a_{4}+a_{1} a_{3} a_{4}+a_{1} a_{2} a_{4}+a_{1} a_{2} a_{3}\right)\left(a_{1}+a_{2}+a_{3}+a_{4}\right)}{\left(a_{1}+a_{2}\right)\left(a_{2}+a_{3}\right)\left(a_{3}+a_{4}\right)\left(a_{4}+a_{1}\right)} .
$$

It is natural to wonder whether formula (4.2) provides an approximation of the determinant in the general case. We have the following asymptotic result:

Theorem 4.1. Let $A_{0}=\operatorname{diag}\left(a_{0}, b_{0}\right)$ be a positive diagonal matrix of $\mathbb{R}^{2 \times 2}$. Let $A_{j}=\operatorname{diag}\left(a_{j}, b_{j}\right), j=1, \ldots, 4$, be four positive diagonal matrices of $\mathbb{R}^{2 \times 2}$, which admit around $A_{0}$ the expansion $A_{j}=A_{0}+\delta B_{j}+\delta^{2} C_{j}+o\left(\delta^{2}\right), j=1, \ldots, 4$. Then, the homogenised matrix $A^{*}$ of the four-phase checkerboard (4.1) $A$ is given by

$$
\operatorname{det} A^{*}=D^{*}+F_{4}\left(A_{0}\right)(E(a)-E(b))+o\left(\delta^{2}\right),
$$

where $D^{*}$ is the determinant given by (4.2), E is given by (4.3) and $F_{4}$ reads as

$$
F_{4}(A):=\frac{16}{\pi^{4}} \sum_{n \in I^{2}} \frac{1}{n_{1}^{2}} \frac{a b^{2}}{a n_{1}^{2}+b n_{2}^{2}},
$$

where $n=\left(n_{1}, n_{2}\right)$ and where the sum is taken over all odd numbers.

Remark 4.2. The difference $E(a)-E(b)$ corresponds to the distance between the two determinants $D^{*}$ and $\tilde{D}^{*}$ obtained as limits by deformation. It is remarkable that, once corrected of this difference scaled by the constant factor $F_{4}\left(A_{0}\right)$, the candidate homogenised determinant $D^{*}$ is valid up to the second order.

There are several examples for which the asymptotic stability condition $E(a)=$ $E(b)$ gives the correct determinant:

- As already mentioned, in the case of an isotropic checkerboard, $E(a)=E(b)$ and $\operatorname{det} A^{*}=D^{*}$.

- In the case of laminates of diagonal matrices, that is, when

$$
\operatorname{det}\left(\begin{array}{ll}
a_{1} & a_{2} \\
a_{4} & a_{3}
\end{array}\right)=\operatorname{det}\left(\begin{array}{ll}
b_{1} & b_{2} \\
b_{4} & b_{3}
\end{array}\right)=0
$$

which implies $E(a)=E(b)=1$, the microstructure $A$ is multipliable (see Proposition 3.4) and thus $\operatorname{det} A^{*}=D^{*}$.

- In the case of a two-phase anisotropic checkerboard structure, the asymptotic stability condition is optimal. Indeed, if $E(a)=E(b)$ then an easy computation yields

$$
\left(\operatorname{det} A_{1}-\operatorname{det} A_{2}\right)\left[\frac{\left(\operatorname{tr} A_{1}\right)^{2}}{\operatorname{det} A_{1}}-\frac{\left(\operatorname{tr} A_{2}\right)^{2}}{\operatorname{det} A_{2}}\right]=0 \quad \text { and } \quad D^{*}=\sqrt{\operatorname{det}\left(A_{1} A_{2}\right)},
$$


whence $\operatorname{det} A^{*}=D^{*}$ by Theorem 2.1 .

The following Theorem (which will proved in the last Section) shows that it is not the case in general for four-phase anisotropic checkerboards:

Theorem 4.3. There exists a four-phase anisotropic checkerboard with $E(a)=$ $E(b)$ and $\operatorname{det} A^{*} \neq D^{*}$.

Remark 4.4. Therefore, contrary to what was obtained for two-phase checkerboards, the second order term $F_{4}\left(A_{0}\right)(E(a)-E(b))$ of expansion (4.4) cannot be used as an indicator of the simplicity or complexity of the formula of the effective determinant. In that sense and in contrast to expansion (2.4), expansion (4.4) is not optimal.

Proof of Theorem 4.1. The proof is similar to that of Theorem 2.1. By the definition of the four-phase checkerboard matrix-valued $A$ defined by (4.1) the Fourier coefficients of the first-order term $B$ in (2.7) with its four phases $B_{j}=\operatorname{diag}\left(\alpha_{j}, \beta_{j}\right)$, $j=1, \ldots, 4$, are given by

$$
B^{n}= \begin{cases}\frac{1}{\pi^{2} n_{1} n_{2}} \operatorname{diag}\left(\alpha_{1}+\alpha_{3}-\alpha_{2}-\alpha-4, \beta_{1}+\beta_{3}-\beta_{2}-\beta-4\right) & n_{1}, n_{2} \in I, \\ \frac{-1}{2 i \pi n_{1}} \operatorname{diag}\left(\alpha_{1}+\alpha_{4}-\alpha_{2}-\alpha-3, \beta_{1}+\beta_{4}-\beta_{2}-\beta-3\right) & n_{1} \in I, n_{2}=0, \\ \frac{-1}{2 i \pi n_{2}} \operatorname{diag}\left(\alpha_{1}+\alpha_{2}-\alpha_{3}-\alpha-4, \beta_{1}+\beta_{2}-\beta_{3}-\beta-4\right) & n_{1}=0, n_{2} \in I,\end{cases}
$$

where $I$ is the set of the odd integers. The correction matrix $M$ defined by $(2.9)$ thus satisfies

$$
\left\{\begin{array}{l}
M_{11}=S\left(\alpha_{1}+\alpha_{3}-\alpha_{2}-\alpha_{4}\right)^{2}+\frac{1}{16 a_{0}}\left(\alpha_{1}+\alpha_{4}-\alpha_{2}-\alpha_{3}\right)^{2}, M_{12}=0 \\
M_{22}=T\left(\beta_{1}+\beta_{3}-\beta_{2}-\beta_{4}\right)^{2}+\frac{1}{16 b_{0}}\left(\beta_{1}+\beta_{2}-\beta_{3}-\beta_{4}\right)^{2}
\end{array}\right.
$$

where $S, T$ are the series

$$
\left\{\begin{array}{l}
S:=\frac{1}{\pi^{4}} \sum_{n \in I^{2}} \frac{1}{n_{2}^{2}} \frac{1}{a_{0} n_{1}^{2}+b_{0} n_{2}^{2}} \\
T:=\frac{1}{\pi^{4}} \sum_{n \in I^{2}} \frac{1}{n_{1}^{2}} \frac{1}{a_{0} n_{1}^{2}+b_{0} n_{2}^{2}}
\end{array} \quad \text { with } \quad a_{0} S+b_{0} T=\frac{1}{16} .\right.
$$

Then, we expand up to second order the determinant of $A^{*}$ using Tartar's formula (2.8) with the correction matrix (4.5), and the expression $D^{*}$ defined by (4.2). After simplifications of formulae by means of Maple we compare the second-order terms of each expansion and we obtain the desired result (4.4).

\section{A high-contrast counter-example}

The following counter-example is based on a factorisation principle introduced by Astala \& Nesi in order to treat anisotropic periodic composites. The principle consists in making a change of variable in which the new conductivity is still periodic (with a new period) but isotropic. The gradient of the change of variable corresponds to the electric field associated with the rescaled conductivity obtained by 
dividing the initial one by the square root of its determinant; so, the rescaled conductivity is still anisotropic but its determinant is equal to 1 . The new effective conductivity (obtained from the new isotropic composite) is deduced from the old one (obtained from the initial anisotropic composite) thanks to a factorisation formula such that the new and old effective determinants are equal. Therefore, the change of variable allows them to pass from an anisotropic periodic composite to an isotropic one without change of the effective determinant.

In the case of a four-phase checkerboard with diagonal conductivity matrices, we construct a change of variable which reduces to a piecewise linear function, linear in each of the regions where the phase is constant. Combining the constraints satisfied by the electric field, the potential and the current at the interfaces between phases (respectively, periodicity, continuity and continuity of the normal derivative) we obtain a linear system in the coefficients of the piecewise linear function. This system has a non-trivial solution if the eight conductivity coefficients (for the four diagonal phases) satisfy a particular condition. When this conditions holds, the change of variable leads to a new periodic composite, with constant coefficients on an irregular but isotropic four-phase checkerboard. We then propose a suitable choice of highcontrast conductivities such that the new effective conductivity satisfies all the constraints, but such that the explicit formula of Section 4 does not hold for the effective determinant.

(a) A result from Astala and Nesi.

This section is devoted to the proof of Theorem 4.3. We will construct a family of microstructures $A_{\tau}^{\varepsilon}, 0<\tau<1$, satisfying the asymptotic stability condition $E(a)=E(b)$, with $E$ is given by (4.3) and such $\operatorname{det} A_{\tau}^{*} \neq D^{*}$ for $\tau$ close enough to 1 . We will rely on the following result of Alessandrini \& Nesi (2001, 2002):

Theorem 5.1 (Alessandrini-Nesi). Let $A$ be a $Y$-periodic matrix-valued function in $\mathcal{M}_{\# Y}(\alpha, \beta)$. Define $\tilde{A}:=A / \sqrt{\operatorname{det} A}$ a.e. in $Y$. Let $\varphi$ be a function in $H_{\mathrm{loc}}^{1}\left(\mathbb{R}^{2}\right)$ such that $\nabla \varphi$ is $Y$-periodic and which solves the equation $\operatorname{div}(\tilde{A} \nabla \varphi)=0$ in $\mathcal{D}^{\prime}\left(\mathbb{R}^{2}\right)$. Assume that the $Y$-averaged value of $\nabla \varphi$ is a non-zero vector. Then, there exists a stream function $\psi$ in $H_{\mathrm{loc}}^{1}\left(\mathbb{R}^{2}\right)$ such that

$$
J \nabla \psi=\tilde{A} \nabla \varphi \quad \text { a.e. in } \mathbb{R}^{2}, \quad \text { with } \quad J:=\left(\begin{array}{cc}
0 & 1 \\
-1 & 0
\end{array}\right)
$$

which solves the conjugate equation $\operatorname{div}(\tilde{A} \nabla \psi)=0$ in $\mathcal{D}^{\prime}\left(\mathbb{R}^{2}\right)$. Moreover, the matrixvalued function $\Phi: y \mapsto(\varphi(y), \psi(y))$ is an homeomorphism from $\mathbb{R}^{2}$ onto $\mathbb{R}^{2}$ and the $Y$-averaged value of $\nabla \Phi$ is an invertible matrix.

We define $A^{\prime}\left(y^{\prime}\right):=\sqrt{\operatorname{det}(A(y))} I_{2}$, where $y^{\prime}:=(\varphi(y), \psi(y))$. By the conditions satisfied by $\varphi$ and $\psi$ the new variable $y^{\prime}$ also reads as

$$
y^{\prime}=\Phi(y)=B y+\Phi_{\#}(y)
$$

where $B$ is a constant invertible matrix and $\Phi_{\#}$ a $Y$-periodic vector-valued function in $H_{\#}^{1}(Y)^{2}$. In the new coordinate system, $A^{\prime}\left(y^{\prime}\right)$ is periodic of period $B Y$ and is isotropic. Then, the following factorisation result is due to Astala \& Nesi: 
Theorem 5.2 (Astala-Nesi). Using the above notations, if $A^{*}$ is the $H$-limit of $A(y)$ and $A^{\prime *}$ the $H$-limit of $A^{\prime}\left(y^{\prime}\right)$, then

$$
\operatorname{det} A^{\prime *}=\operatorname{det} A^{*} .
$$

For the reader convenience we just give the idea of the proof following Milton's approach as described in Sections 8.5 and 8.6 of Milton (2002):

Proof. On the one hand, using the definition of $\Phi$ in terms of $\varphi$ and $\psi$ yields

$$
A^{\prime}\left(y^{\prime}\right)=\frac{1}{\operatorname{det}(\nabla \Phi(y))} \nabla \Phi(y) A(y) \nabla \Phi^{T}(y), \quad \nabla \Phi(y):=\left(\begin{array}{cc}
\frac{\partial \varphi}{\partial y_{1}}(y) & \frac{\partial \varphi}{\partial y_{2}}(y) \\
\frac{\partial \psi}{\partial y_{1}}(y) & \frac{\partial \psi}{\partial y_{2}}(y)
\end{array}\right) .
$$

One the other hand, the curvilinear form (5.1) of the change of variable $\Phi$ leads to $A^{\prime *}=\frac{1}{\operatorname{det} B} B A^{*} B^{T}$, which in particular implies (5.2).

(b) Application to the four-phase anisotropic checkerboard.

In order to use the result of Astala \& Nesi, we construct a piecewise linear change of variable $y^{\prime}=(\varphi(y), \psi(y))$ satisfying the conditions given in Theorem 5.1, i.e., for $y=\left(y_{1}, y_{2}\right) \in Y_{i}, i=1, \ldots, 4, \varphi(y)=\alpha_{i} y_{1}+\beta_{i} y_{2}+c_{i}$ and $\psi(y)=\gamma_{i} y_{1}+\delta_{i} y_{2}+d_{i}$. Functions $\varphi$ and $\psi$ are continuous and periodic, which yields 16 equations at the

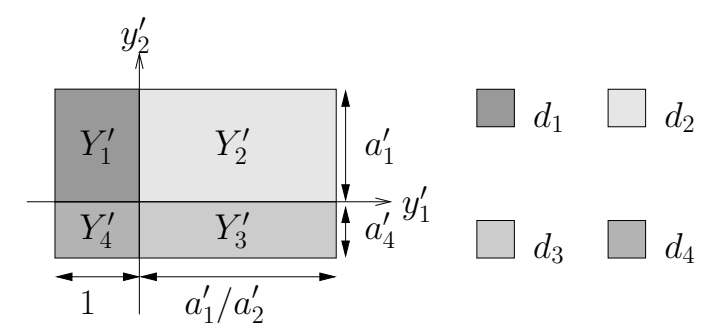

Figure 2. The new period cell $Y^{\prime}$, and the microstructure $A^{\prime}\left(y^{\prime}\right)$. Here, $a_{i}^{\prime}=\sqrt{a_{i} / b_{i}}$ and $d_{i}=\sqrt{a_{i} b_{i}}$ for $i=1, \ldots, 4$.

interfaces between the quadrants of $Y$, repeated periodically. The flux condition $J \nabla \psi=\tilde{A} \nabla \varphi$ provides another 8 identities. It is convenient to write

$$
\tilde{A}=\sum_{i=1}^{4} 1_{Y_{i}} \operatorname{diag}\left(a_{i}^{\prime}, 1 / a_{i}^{\prime}\right), \quad \text { where } \quad a_{i}^{\prime}:=\sqrt{\frac{a_{i}}{b_{i}}} .
$$

If $a_{1}^{\prime} a_{3}^{\prime} \neq a_{2}^{\prime} a_{4}^{\prime}$, these 24 equations imply $\alpha_{i}=\beta_{i}=0$ for $i=1, \ldots, 4$, violating the assumption of Theorem 5.1., i.e. $\int_{Y} \nabla \varphi \neq 0$. We obtain on $Y_{1}$ and $Y_{2}$

$$
y^{\prime}=\left(\begin{array}{cc}
\alpha_{i} & \beta_{i} \\
\frac{\beta_{i}}{a_{i}^{\prime}} & -a_{i}^{\prime} \alpha_{i}
\end{array}\right) y+\mathrm{cst} \quad \text { for } y \in Y_{i}, i=1,2 .
$$

The columns of both matrices are orthogonal thus $y \mapsto y^{\prime}$ is an orthogonal change of variable. Furthermore, the continuity along the interface yields $\beta_{1}=\beta_{2}$ and 
$a_{1}^{\prime} \alpha_{1}=a_{2}^{\prime} \alpha_{2}$. Therefore, $Y_{1}^{\prime}$ and $Y_{2}^{\prime}$ are two rectangles with a common side. The quadrant $Y_{1}^{\prime}$ has length

$$
l_{1}^{\prime}=\sqrt{\alpha_{1}^{2}+\left(\frac{\beta_{1}}{a_{1}^{\prime}}\right)^{2}} \quad \text { and } \quad a_{1}^{\prime} l_{1}^{\prime}=\sqrt{\beta_{1}^{2}+\left(a_{1}^{\prime} \alpha_{1}\right)^{2}},
$$

whereas $Y_{2}^{\prime}$ has length

$$
l_{2}^{\prime}=\sqrt{\alpha_{2}^{2}+\left(\frac{\beta_{2}}{a_{2}^{\prime}}\right)^{2}}=\frac{a_{1}^{\prime}}{a_{2}^{\prime}} l_{1}^{\prime} \quad \text { and } \quad a_{2}^{\prime} l_{2}^{\prime}=a_{1}^{\prime} l_{1}^{\prime} .
$$

Performing the same analysis on the other adjacent quadrants, we obtain that $Y$ maps onto $Y^{\prime}$ the rectangle represented on Figure 2 on page 16. Since $l_{1}^{\prime}$ is an arbitrary parameter, we set $l_{1}^{\prime}=1$. The corresponding matrix

$$
A^{\prime}=\sum_{i=1}^{4} \sqrt{a_{i} b_{i}} 1_{Y_{i}^{\prime}} \text { for } i=1, \ldots, 4,
$$

is represented on Figure 2 on page 16. We can check that the $Y$-averaged valued of $\nabla \varphi$ is also a non-zero vector. Therefore, according to Theorem $5.1 A^{\prime *}$ and $A^{*}$ have the same determinant.

Remark 5.3. In the case when all four phases have equal area, i.e., $\left|Y_{1}^{\prime}\right|=\left|Y_{2}^{\prime}\right|=$ $\left|Y_{3}^{\prime}\right|=\left|Y_{4}^{\prime}\right|$, the matrix-valued function $A^{\prime}$ is an isotropic four-phase checkerboard; its homogenised matrix is given in Craster \& Obnosov (2001). This happens when $a_{1}^{\prime}=a_{2}^{\prime}=a_{3}^{\prime}=a_{4}^{\prime}$, that is, when $a_{i}=\alpha b_{i}$ for all $i=1, \ldots, 4$ and for some positive $\alpha$. In this case, the simpler (linear) change of variable $y^{\prime}=B y$, with $B:=$ $\operatorname{diag}(1, \sqrt{\alpha})$, leads directly to the isotropic checkerboard thanks to Proposition 3.3. Using the Craster-Obnosov result we also obtain

$$
\operatorname{det} A^{\prime *}=\alpha\left(\frac{a_{2} a_{3} a_{4}+a_{1} a_{3} a_{4}+a_{1} a_{2} a_{4}+a_{1} a_{2} a_{3}}{a_{1}+a_{2}+a_{3}+a_{4}}\right)=D^{*} .
$$

(c) Proof of Theorem 4.3.

Consider the four-phase checkerboard (4.1) given by

$$
A_{1}=\operatorname{diag}\left(a_{1}, b_{1}\right), \quad A_{3}=\operatorname{diag}\left(b_{1}, a_{1}\right), \quad A_{2}=a_{2} I_{2} \quad \text { and } \quad A_{4}=\sqrt{a_{1} b_{1}} I_{2} .
$$

Using the same notations as above,

$$
a_{1}^{\prime}=\sqrt{\frac{a_{1}}{b_{1}}}, \quad a_{2}^{\prime}=1, \quad a_{3}^{\prime}=\sqrt{\frac{b_{1}}{a_{1}}} \quad \text { and } \quad a_{4}^{\prime}=1 .
$$

We do have $a_{1}^{\prime} a_{3}^{\prime}=1=a_{2}^{\prime} a_{4}^{\prime}$. Therefore, $\operatorname{det} A^{*}=\operatorname{det} A^{\prime *}$, where

$$
A^{\prime}=d_{1}\left(1_{Y_{1}^{\prime}}+1_{Y_{3}^{\prime}}+1_{Y_{4}^{\prime}}\right)+a_{2} 1_{Y_{2}^{\prime}}, \quad \text { with } \quad d_{1}=\sqrt{a_{1} b_{1}} .
$$

The domain $Y^{\prime}$ is a square of side $1+a_{1}^{\prime}$, and the quadrant $Y_{2}^{\prime}$ is also a square of side $a_{1}^{\prime}=\sqrt{a_{1} / b_{1}}$. Since $\left(b_{1}, b_{2}, b_{3}, b_{4}\right)=\left(a_{3}, a_{2}, a_{1}, a_{4}\right)$, we have $E(a)=E(b)$. In this case, formula (4.4) reads as

$$
\operatorname{det} A^{*}=D^{*}+o\left(\delta^{2}\right), \quad D^{*}=d_{1}^{2} \frac{\left(d_{1}+a_{2} a_{1}^{\prime}\right)\left(d_{1} a_{1}^{\prime}+a_{2}\left(1+a_{1}^{\prime}+a_{1}^{\prime 2}\right)\right)}{\left(a_{2}+d_{1} a_{1}^{\prime}\right)\left(d_{1}+a_{1}^{\prime}\left(a_{2}+d_{1}+d_{1} a_{1}^{\prime}\right)\right)} .
$$


We will prove that $\operatorname{det} A^{*}$ cannot always be equal to $D^{*}$. First, note that we can transform $Y^{\prime}$ to a square of side 1 , by a homothetic transformation (which does not change the value of the homogenised matrix). The new geometry is represented.

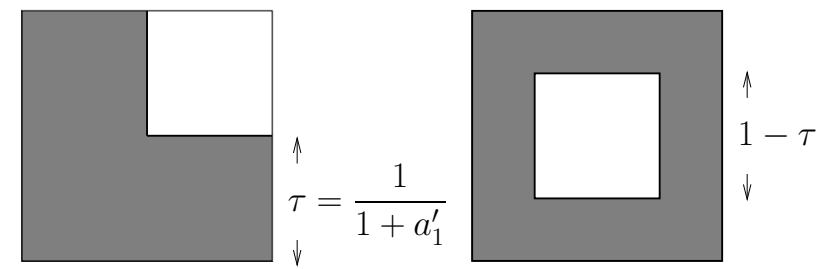

On the one hand, by letting $a_{2}$ tend to zero we obtain a structure where subsquare $Y_{2}^{\prime}$ is not conducting. Note that the homogenised matrix of the structure obtained when $a_{2}$ is set to zero is same as the one obtained passing to the limit when $a_{2}$ tends to zero. When the volume fraction is small, the formula for $A^{*}$ is that of a "Virtual Mass". Jikov et al. (1994, pp. 106-107) proved that

$$
A^{*}=d_{1} I_{2}+d_{1}(1-\tau)^{2} \mathcal{L}+o\left((1-\tau)^{2}\right)
$$

where $\mathcal{L}$ is a symmetric positive definite matrix.

On the other hand, when $a_{2}$ tends to zero the conjectured determinant $D^{*}$ defined by (5.3) satisfies

$$
D^{*}=\frac{d_{1}^{2}}{1+a_{1}^{\prime}+a_{1}^{\prime 2}}=d_{1}^{2} \tau+o(1-\tau) .
$$

Therefore, $\operatorname{det} A^{*} \neq D^{*}$ for $\tau$ close to 1 .

\section{Conclusion}

This contribution points out that the effective properties of an anisotropic fourphase periodic checkerboard can be partially but explicitly attained through the expansion of the effective determinant. Indeed, for a two-phase checkerboard a second-order expansion formula provides an explicit approximation of the effective determinant which is valid if the conductivity matrices commute and have a weak contrast. A remarkable fact is that the exact value for the effective determinant is obtained when the second-order term of the expansion vanishes. In that sense, this expansion is somewhat optimal.

In the more general case of a four-phase checkerboard the stability property introduced in Section 3 holds true for any isotropic four-phase rectangular checkerboard thanks to the Craster-Obnosov formula. This property allows us to derive an explicit second-order expansion for a four-phase checkerboard with diagonal conductivity matrices. Unfortunately, in contrast to the two-phase checkerboard case, the cancellation of the second-order term of the expansion does not imply that the exact value of the effective determinant is given by the expansion, as it is shown by the counter-example of Section 5.

The counter-example is interesting in itself since it provides an application of a nice factorisation principle for anisotropic periodic composites, due to Astala and Nesi. In that example, an explicit construction of piecewise linear functions in each 
phase allows us to transform an anisotropic four-phase square checkerboard into an irregular but isotropic one with the same effective determinant.

Amongst the initial motivations for this work was the desire to evaluate an automatic formula generation algorithm. Both positive and negative results presented in this article can be exploited to this end. Moreover, it could be used to design (analytical) benchmarks for anisotropic homogenisation numerical codes. Alternatively, the approach based on the stability property is not restricted to four-phase checkerboards. It could be exploited to obtain explicit expansion formulae of the effective determinant for other periodic composites.

The authors wish to thank V. Nesi for a stimulating discussion and relevant comments concerning the derivation of the counter-example. The authors were partly supported by ACINIM 2003-45.

\section{References}

Allaire, G. 1992 Homogenization and two-scale convergence. SIAM J. Math. Anal. 23, no. $6,1482-1518$.

Alessandrini, G., Nesi, V. 2001 Univalent $\sigma$-harmonic mappings. Arch. Rat. Mech. Anal. 158, 155-171.

Alessandrini, G., Nesi, V. 2002 Univalent $\sigma$-harmonic mappings: applications to composites. ESAIM Control Optim. Calc. Var. 7, 379-406.

Astala, K., Nesi, V. Area distortion for periodic quasiconformal mappings. Private communication, partially written in Milton (2002) Section 8.6, 152-155.

Bensoussan, A., Lions, J.-L., Papanicolaou, G. C. 1978 Asymptotic analysis for periodic structures. Amsterdam: North-Holland Publishing Co.

Berdichevski, V. L. 1985 The thermal conductivity of chess structures. VestnovMosk. Univ. Mat. Mech. 40, 56-63.

Craster, R. V., Obnosov, Y. V. 2001 Four-phase checkerboard composites. SIAM J. Appl. Math. 61, no. 6, 1839-1856.

Dykhne, A. M. 1970 Conductivity of a two-dimensional two-phase system. A. Nauk. SSSR 59, 110-115 (English transl. in Soviet Physics JETP 1971, 32, 63-65).

Fabre, S., Mossino, J., $1998 \mathrm{H}$-convergence of multiplicable matrices. Calc. Var. Part. Diff. Eqns 7 no. 2, 125-139.

Francfort, G., Murat, F. 1987 Optimal bounds for conduction in two-dimensional, twophase, anisotropic media. In Non-Classical Continuum Mechanics: Proceedings of the London Mathematical Society Symposium, Durham, July 1986 (eds R. J. Knops \& A. A. Lacey), pp. 197-212. Cambridge University Press.

Golden, K, Milton, G. W. 1990 Representations for conductivity functions of multicomponent composites. Comm. Pure Appl. Math 43, 647-671.

Jikov, V. V., Kozlov, S. M., Oleinik, O. A. 1994 Homogenization of Differential Operators and Integral Functionals. New York:Springer.

Keller, J. B. 1964 A theorem on the conductivity of a composite medium. J. Math. Phys. 5 no. 4, 548-549.

Maxwell, J. C. 1904 A treatise on electricity and magnetism. Oxford University Press.

Mendelson, K. S. 1975 A theorem on the effective conductivity of a two-dimensional heterogeneous medium. J. Appl. Physics 46 no. 11, 4740-4741.

Michel, J. C., Moulinec, H., Suquet, P. 1999 Effective properties of composite materials with periodic microstructure: A computational approach. Comput. Methods Appl. Mech. Eng. 172 no. 1-4, 109-143. 
Milton, G. W. 2001 Proof of a conjecture on the conductivity of checkerboards. J. Math. Phys. 42 no. 10, 4873-4882.

Milton, G. W. 2002 The theory of composites. Cambridge Monographs on Applied and Computational Mathematics. Cambridge University Press.

Mityushev, V. V. 1995 Transport properties of double periodic arrays of circular cylinders. Z. Angew. Math. Mech. 77, 115-120.

Mortola, S., Steffé, S. 1985 A two-dimensional homogenization problem. Atti Accad. Naz. Lincei Rend. Cl. Sci. Fis. Mat. Natur. 78 no. 3, 77-82.

Murat, F., Tartar, L. 1978 H-convergence. Séminaire d'Analyse Fonctionnelle et Numérique de l'Université d'Alger mimeographed notes.

Murat, F., Tartar, L. 1997 H-convergence.In Topics in the mathematical modelling of composite materials pp.21-43. Progr. Nonlinear Differential Equations Appl., vol. 31. Boston: Birkhäuser.

Nevard, J., Keller, J. B. 1985 Reciprocal relations for effective conductivities of anisotropic media. J. Math. Phys. 26, 2761-2765.

Obnosov, Y. V. 1999 Periodic heterogeneous structures: new explicit solutions and effective characteristics of refraction of an imposed field. SIAM J. Appl. Math. 59, 1267-1287.

Rayleigh, J. W. 1892 On the influence of obstacles arranged in rectangular order upon the properties of the medium. Phil. Mag. 34, 481-502.

Tartar, L. 1990 H-measures, a new approach for studying homogenization, oscillations and concentration effects in partial differential equations. Proc. Roy. Soc. Edinburgh 115A, 193-230.

Tartar, L. 2000 An introduction to the homogenization method in optimal design. Springer Lecture Notes in Mathematics, no. 1740, pp. 47-156.

Torquato, S., Kim, I. C., Cule, D. 1999 Effective Conductivity, Dielectric Constant, and Diffusion Coefficient of Digitized Composite Media via First-Passage-Time-Equations. J. Appl. Physics 85, 1560-1571. 